\title{
Effect of Mixing and Other Operating Parameters in Sol-Gel Processes
}

\author{
Daniele L. Marchisio, ${ }^{*}{ }^{\dagger}$ Federica Omegna, ${ }^{\dagger}$ Antonello A. Barresi, ${ }^{\dagger}$ and Paul Bowen ${ }^{\ddagger}$ \\ Dipartimento di Scienza dei Materiali e Ingegneria Chimica, Politecnico di Torino, C.so Duca degli Abruzzi \\ 24, 10129 Torino, Italy, and Laboratoire de technologie des poudres, Ecole Polytechnique Fédérale de \\ Lausanne, MX-D 336 Station 12, CH-1015 Lausanne, Switzerland
}

In this work the effect of mixing on a sol-gel process is quantitatively investigated. Titanium dioxide synthesis from titanium tetra-isopropoxide is used as a test reaction. Solutions of titanium alkoxide in isopropyl alcohol and water in isopropyl alcohol are mixed in a special mixing device (i.e., vortex reactor) at different mixing rates, and the effect of mixing is quantified and compared with the effect of the other relevant operating parameters, namely the water to alkoxide, acid to alkoxide ratios, and alkoxide initial concentration. Dynamic light scattering, specific surface area measurement through nitrogen adsorption, X-ray diffraction, and field emission scanning electron microscopy are employed to determine particle size distribution, morphology, and crystallite size of the different particulate products (i.e., sols, gels, powders) obtained before and after thermal treatments under different synthesis conditions. A factorial design is used to plan the experimental campaign and results show that the role of mixing cannot be neglected. Moreover results show that mixing can be actively used to control the final product characteristics and must be taken into account when a process is transferred from the laboratory to the industrial scale. Eventually a scale-up criterion based on our previous work will be discussed.

\section{Introduction}

Sol-gel processes are used to prepare a wide variety of materials and can be divided into two categories: aqueous-based processes that start from solutions of a salt and alcohol-based processes that usually start from a metal alkoxide. In this work we will focus only on the second category; however, our conclusions hold in a much more general contest.

In all these processes it is necessary to mix two or more solutions to start the chemical reactions involved, namely hydrolysis and condensation. During hydrolysis the metal alkoxide $\mathrm{M}-\mathrm{OR}$ is broken down by water molecules, and one or more alkoxide groups are replaced by hydroxide groups. During condensation water or alcohol molecules are eliminated through different mechanisms (i.e., alkoxolation, oxolation, olation, polycondensation, etc.) and oxygen bridges are formed between metal atoms. The process is also described in terms of a particle formation step, controlled by nucleation and molecular growth, and a subsequent agglomeration step, where already formed particles collide and stick together. The relative rates of these processes are very important since they determine the final product characteristics, such as particle size distribution (PSD) and morphology, as well as the overall particulate structure (e.g., sol versus gel).

For example, it is a well established rule of thumb ${ }^{1}$ that slow hydrolysis and slow condensation rates usually result in the production of stable monodispersed sols, that mainly nucleate and grow by continuous molecular processes. On the contrary fast hydrolysis and slow condensation may result in rapid particle formation (through rapid particle nucleation and growth) and subsequent (although limited) agglomeration, forming polymeric sols, namely particulate systems constituted by very sparse aggregates or clusters and characterized by quite small fractal dimensions. Fast hydrolysis and fast condensation result in the almost instantaneous formation of a gel, due to rapid

* To whom correspondence should be addressed. E-mail: daniele. marchisio@ polito.it. Tel.: 0039-0115644622. Fax: 0039-0115644699.

${ }^{\dagger}$ Politecnico di Torino.

* Ecole Polytechnique Fédérale de Lausanne. nucleation, growth, and agglomeration and eventually slow hydrolysis and fast condensation result in controlled precipitation. The actual hydrolysis and condensation rates depend on many parameters, such as the nature of the alkoxide group and of the alcohol used as solvent, the initial water and metal alkoxide concentrations, and the concentration of hydrogen ions and other additives.

The nature of the alkoxide group affects the hydrolysis and condensation rates. In fact, it is well-known that the hydrolysis rate of a metal alkoxide decreases when increasing the size of the alkyl group (e.g., ethoxide, propoxide, butoxide) as a consequence of the positive partial charge of the metal atom, which decreases with alkyl chain length, as shown by many authors. $^{2-8}$

Metal alkoxides are often dissolved in organic solvents before hydrolysis is performed. The most often used solvents are parent alcohols, which have the same number of carbon atoms as the metal alkoxide. However, it should be noted that solvents are often not chemically inert toward metal alkoxides and that their reactivity can be easily modified by changing the solvent. ${ }^{8-10}$

Hydrolysis and condensation rates are also largely affected by the water to alkoxide initial concentration ratio ${ }^{10,11}$ (also referred to as hydrolysis ratio) that in turn determines the final particle size. Two major domains are usually detected, one at low water contents, where an increase in the hydrolysis ratio causes a reduction of the particle size, and another one at high water contents, where an increase in the hydrolysis ratio causes an increase in the particle size. Usually these two regions are related to the number of water molecules per metal alkoxide molecule needed for complete hydrolysis (equal to four for titanium alkoxides). At amounts of water much lower than the stoichiometric value, hydrolysis is slow with respect to condensation, and the excess of titanium alkoxide in the solvent favors the development of $\mathrm{Ti}-\mathrm{O}-\mathrm{Ti}$ chains through alcoxolation, resulting in relatively big particles. When the hydrolysis ratio is increased (but kept below the stoichiometric value) the formation of completely hydrolyzed products is favored and the relative contribution of alcoxolation is reduced. Larger amounts 
of water suppresses the development of $\mathrm{Ti}-\mathrm{O}-\mathrm{Ti}$ chains also by oxolation, resulting in smaller particles. A further increase in the water content at about the stoichiometric value results in a local excess of water that favors the coordination of water to $\mathrm{Ti}(\mathrm{OH})_{4}$ (i.e., solvation) resulting in more reactive species that can again contribute to the development of new $\mathrm{Ti}-\mathrm{O}-\mathrm{Ti}$ chains through olation, yielding to even bigger particles.

When metal alkoxides are used as precursors, it has been traditionally assumed that condensation is much slower than hydrolysis; however, quite recently it was shown that the rates of these processes are actually very close. Kinetic studies ${ }^{12}$ of hydrolysis of titanium and zirconium alkoxides have shown that both hydrolysis and condensation are quite fast, and that condensation occurs after only 25 to $50 \%$ of the original alkoxide groups are substituted by hydroxides (under the experimental conditions investigated in the cited work).

The course of hydrolysis and condensation is also affected by the presence of catalysts or inhibitors, such as inorganic acids or complexing ligands. For example, hydrogen ions are believed to favor hydrolysis, by protonation of the alkoxy groups, and to act as inhibitors for condensation. ${ }^{7}$

It has also been reported in the literature that mixing has an impact on sol-gel processes; however, its effect has never been quantitatively determined and comprehensively investigated. The explanation of this effect lies in the fact that chemical reactions occur at the molecular level, and therefore they happen in a homogeneous environment only if the rate at which reactants are mixed (at the molecular level) is much higher than the characteristic chemical reaction rate. On the contrary, when the involved reactions are very fast, such as hydrolysis and condensation in many practical cases, they might start before mixing is complete, and therefore in these cases mixing determines the local conditions under which these fast chemical reactions actually occur. It is very important to quantify mixing effects, because if it is quite easy to control the mixing efficiency at the laboratory scale, this is not often the case when a process is transferred from the laboratory to the industrial scale where the objectives are to reduce undesirable high operating costs and product quality variations and to increase low production rates. The effect of mixing is also important because in continuous apparatuses (e.g., stirrer tanks) it controls residence time distribution and macro-mixing features in the reactor.

Some of these issues have been studied in the past; different reactor configurations were employed ${ }^{13-15}$ and the effect of different mixing conditions investigated. ${ }^{16}$ Only recently some detailed studies on the effect of mixing on particle formation processes have appeared in the literature. ${ }^{17,18}$ Results show that to properly control the final particle characteristics it is essential to control mixing, and this can be done by employing various types of reactors, such as a concentric microreactor, ${ }^{19}$ a rotating spinning disk reactor, ${ }^{20}$ a confined impinging jet reactor, ${ }^{21}$ or a vortex reactor. ${ }^{22}$ Moreover only in recent years sophisticated and reliable computational tools, such as computational fluid dynamics (CFD) have been developed. As shown by many authors, CFD can be used to model and simulate the particle formation process, to quantify the effect of each of the competing phenomena, and to calculate the characteristics mixing and particle formation time-scales that can then be used for process scale-up. Different approaches exist, and in this work the methodology developed in our previous work will be used. ${ }^{23,24}$

In this study the effect of mixing on a sol-gel process is investigated from the experimental and modeling viewpoints. The synthesis of titanium dioxide from titanium tetra-isopro- poxide and water in isopropyl alcohol is employed as a test reaction. Different mixing conditions are investigated by resorting to a special passive mixer called a vortex reactor (VR). In this device the characteristic mixing time can be controlled by varying the flow rates of the inlet solutions, as supported by CFD simulations. The effect of mixing is studied and compared with the effect of the other relevant parameters usually considered during the optimization of particle synthesis protocols, such as water to alkoxide and acid to alkoxide concentration ratios and alkoxide initial concentration.

A factorial design is used to plan the experimental campaign and to statistically analyze the collected experimental data. The range of variation of each individual operating variable was identified after an explorative experimental campaign. The experimental characterization of the different particulate products obtained (i.e., sols, gels, powders) was carried out by using standard techniques (i.e., dynamic light scattering, specific surface area measurements, X-ray diffraction, and field emission scanning electron microscopy).

\section{Materials, Experimental Details, and Operating Conditions}

Titanium tetra-isopropoxide (TTIP) was used as a precursor in this work, because of its very rapid hydrolysis kinetics. Two solutions of TTIP in isopropyl alcohol and of water in isopropyl alcohol were prepared separately under nitrogen flux to control the alkoxide reactivity with humidity. Hydrochloric acid $(\mathrm{HCl})$ was added at different initial concentrations to the second solution as a hydrolysis catalyst and deagglomeration agent. Glass vials were rinsed with isopropyl alcohol and dried at ca. $120^{\circ} \mathrm{C}$ before use. Solutions were prepared by using reagent grade reactants (Sigma-Aldrich) and ultrafiltrated water from reverse osmosis (Millipore Milli-Q RG).

The two solutions of TTIP and water in isopropyl alcohol were mixed in a VR. The VR is a passive mixer characterized by a small volume (about $0.5 \mathrm{~mL}$ ), whose mixing time can be tuned by changing the flow rates of the inlet solutions, as will become clearer in the next section. The geometrical details of this reactor are reported in Figure 1. To investigate the effect of mixing in a wide range of operating conditions, the VR was connected to an infusion pump (KDS200 syringe pumps by KD Scientific) and inlet flow rates could be manipulated from 3 to $120 \mathrm{~mL} / \mathrm{min}$. For higher flow rates a different feeding system was employed, and the two solutions were stored in two small identical vessels, then pressurized at 3 bar with analytical grade nitrogen; the inlet flow rates were manipulated from 80 to 300 $\mathrm{mL} / \mathrm{min}$ with two rotameters. When the inlet flow rates (FR) of the two reactant solutions are varied from 3 to $300 \mathrm{~mL} / \mathrm{min}$, the mean residence time in the mixer changes from about 5 to $0.05 \mathrm{~s}$ and the flow field is completely modified, moving from low turbulence and very poor mixing performances to highly turbulent conditions with very rapid and efficient mixing dynamics.

Equal volumes of reactant solutions (i.e., $100 \mathrm{~mL}$ ) were mixed at equal flow rates at $30{ }^{\circ} \mathrm{C}$, and then for both configurations the solutions exiting the reactor were collected in a small vessel thermostatted at $30{ }^{\circ} \mathrm{C}$ and gently stirred. The PSD of the sol obtained was measured after exactly 2 min by dynamic light scattering (DLS) with Malvern Nano ZS90 providing reliable information in the size range from 1 to $6000 \mathrm{~nm}$; the PSD was then used to determine the mean particle size $\left(d_{\text {mean }}\right)$. The same instrument was also used to measure the $\zeta$ potential of some selected samples. As already reported, the solutions were then gently stirred, and the evolution of PSD was tracked until a 


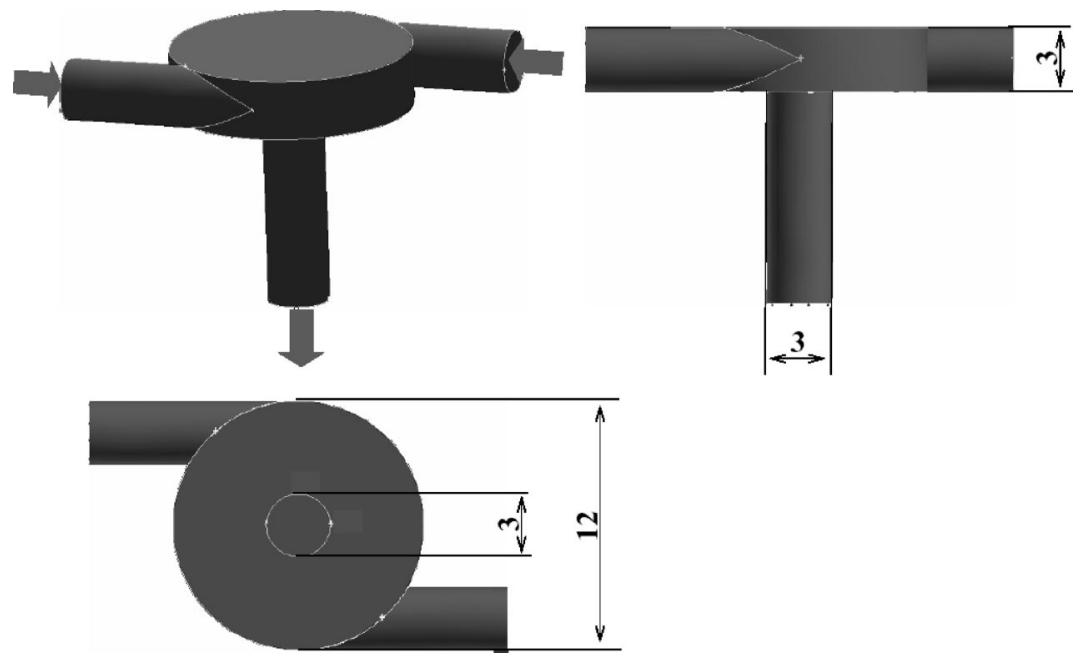

Figure 1. Sketch of the vortex reactor; quotes are in millimeters.

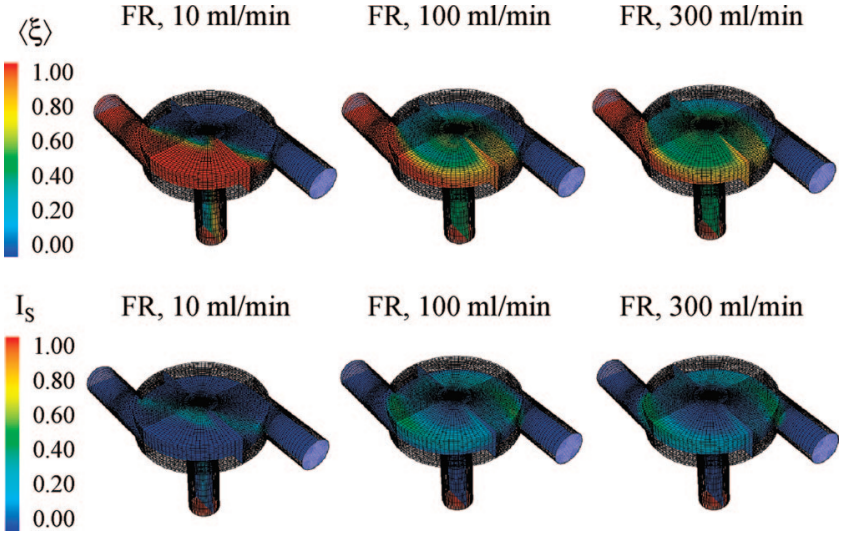

Figure 2. Contour plot of inert tracer averaged-concentration (top) and intensity of segregation (bottom) at different flow rates.

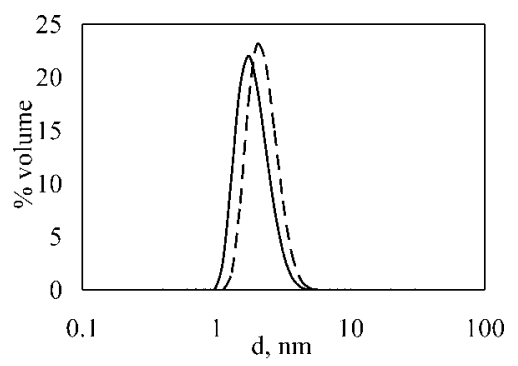

Figure 3. Volume PSDs for the $\mathrm{TiO}_{2}$ sols 2 min after mixing under the operating conditions of experiment 11 of Table 1 . The continuous and dashed lines refer to the same experiment repeated twice.

steady state was reached. The solutions were then filtered with a microfiltration system (Sartorius P.47, $200 \mathrm{~mL}$ ) using a 0.1 $\mu \mathrm{m}$ membrane, dried at $120^{\circ} \mathrm{C}$, washed with a solution of $0.02 \%$ in weight of isopropyl alcohol and polyacrylic acid (PAA 2000, Aldrich), and after 15 min of ultrasonic treatment the suspension was filtered again. The resulting powders were eventually calcined at $400{ }^{\circ} \mathrm{C}$ for $2 \mathrm{~h}$.

The dried (and calcined) powders were further characterized in order to determine the specific surface area $\left(\mathrm{SSA}, \mathrm{m}^{2} / \mathrm{g}\right)$ by nitrogen adsorption (Micrometrics Gemini 2375) by using the Brunauer-Emmett-Teller (BET) theory and the equivalent spherical diameter $\left(d_{\mathrm{BET}}\right)$ was calculated. Samples were also characterized by using X-ray diffraction (XRD, PhiliphsPW3830) in order to determine the different polymorphs (i.e., rutile versus anatase).
Table 1. Operating Conditions Used in the 16 Runs for the DOE

\begin{tabular}{lcclc}
\hline ID & [TTIP], mol/L & $W$ & $H$ & FR, mL/min \\
\hline 1 & 0.5 & 2 & 0.25 & 3 \\
2 & 0.5 & 4 & 0.25 & 3 \\
3 & 1 & 2 & 0.25 & 3 \\
4 & 1 & 4 & 0.25 & 3 \\
5 & 0.5 & 2 & 0.5 & 3 \\
6 & 0.5 & 4 & 0.5 & 3 \\
7 & 1 & 2 & 0.5 & 3 \\
8 & 1 & 4 & 0.5 & 3 \\
9 & 0.5 & 2 & 0.25 & 110 \\
10 & 0.5 & 4 & 0.25 & 110 \\
11 & 1 & 2 & 0.25 & 110 \\
12 & 1 & 4 & 0.25 & 110 \\
13 & 0.5 & 2 & 0.5 & 110 \\
14 & 0.5 & 4 & 0.5 & 110 \\
15 & 1 & 2 & 0.5 & 110 \\
16 & 1 & 4 & 0.5 & 110
\end{tabular}

${ }^{a}$ Low and high levels for the initial alkoxide concentration [TTIP], the hydrolysis ratio $W$, the acid to alkoxide ratio $H$, and the flow rate (FR) with which the reactant solutions are fed to the VR. Each experiment is repeated twice.
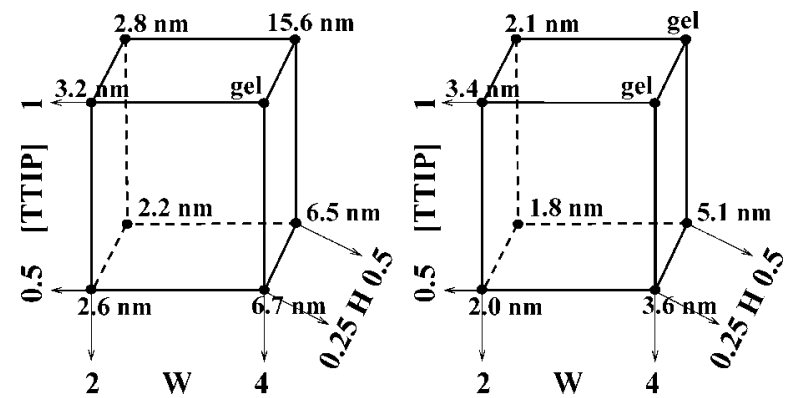

Figure 4. $\mathrm{TiO}_{2}$ mean particle size $\left(d_{\text {mean }}, \mathrm{nm}\right) 2 \mathrm{~min}$ after mixing for the experimental conditions reported in Table 1 (left side FR $=3 \mathrm{~mL} / \mathrm{min}$ and right side $\mathrm{FR}=110 \mathrm{~mL} / \mathrm{min}$ ).

Particle morphology was analyzed by scanning electron microscopy (SEM) and by field emission scanning electron microscopy (FE-SEM). Samples were prepared for observation by depositing a drop of the suspension or of the dried powder on a TEM grid or an a SEM sample stage. SEM and FE-SEM analyses were performed on the dried and calcined powders.

The effect of several operating conditions on the final characteristics of the sol/gel obtained and of the dried and calcined powders was investigated. In particular the effect of the initial molar concentration of TTIP in the isopropyl alcohol solution [TTIP] was studied in the range between 0.5 and 1.0 

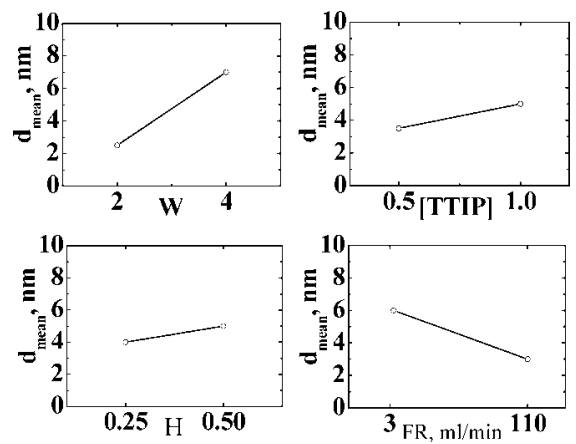

Figure 5. Main effect of the four investigated parameters $W$, [TTIP], $H$, and $\mathrm{FR}$ on $\mathrm{TiO}_{2}$ mean particle size $\left(d_{\text {mean }}\right) 2$ min after mixing.

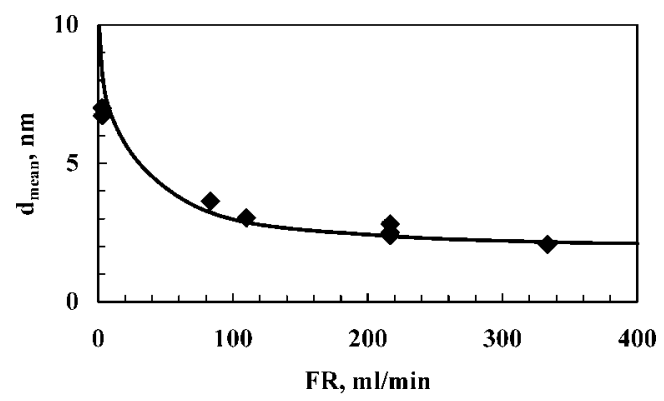

Figure 6. $\mathrm{TiO}_{2}$ mean particle size $\left(d_{\text {mean }}\right) 2$ min after mixing at different FR values for $[$ TTIP] $=0.5 \mathrm{~mol} / \mathrm{L}, W=4$, and $H=0.25$.

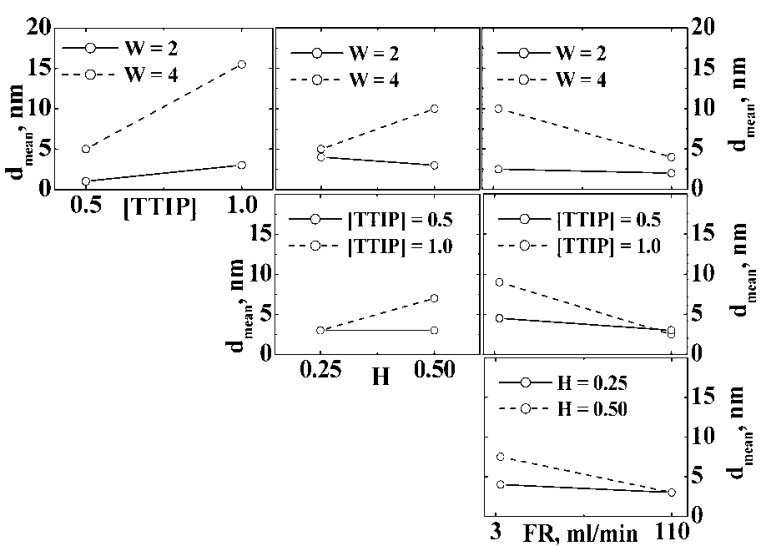

Figure 7. Interaction plots reporting the effect on $\mathrm{TiO}_{2}$ mean particle size $\left(d_{\text {mean }}\right) 2$ min after mixing of each investigated parameter when the other three parameters are kept at low and high levels.

$\mathrm{mol} / \mathrm{L}$, whereas the effect of the final water to TTIP molar concentration ratio $W=\left[\mathrm{H}_{2} \mathrm{O}\right] /[\mathrm{TTIP}]$ was investigated in the range between 2 and 4 . Also the effect of hydrochloric acid was explored varying the acid to TTIP molar concentration ratio $H=[\mathrm{HCl}] /[\mathrm{TTIP}]$ between 0.25 and 0.5 . The feeding flow rates (FR) of the reactant solutions in the VR were varied between 3 and $300 \mathrm{~mL} / \mathrm{min}$. The combined effect of these operating parameters and their relevant interactions were quantitatively investigated through a design of experiments (DOE). The DOE was carried out by considering two levels for the four different parameters investigated resulting in a $2^{4}$ factorial design. The final set of the planned experiments is reported in Table 1. Each experiment was repeated twice to asses the accuracy and reproducibility of the synthesis method and the final 32 runs were performed in randomized order.

The experimental results were then analyzed using the socalled analysis of variance (ANOVA) or regression analysis. ${ }^{25,26}$ Through this analysis it is possible to estimate the main effect

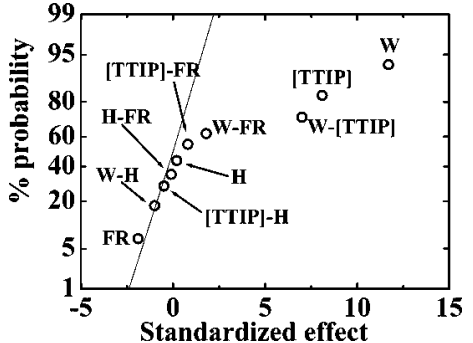

Figure 8. Probability plot for the standardized effects of the investigated parameters and their interactions on $\mathrm{TiO}_{2}$ mean particle size $\left(d_{\text {mean }}\right) 2$ min after mixing; the straight line represents the normal probability distribution.

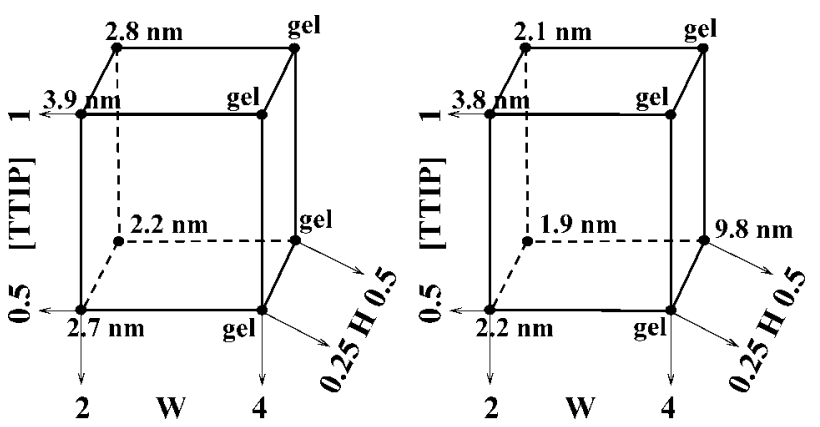

Figure 9. $\mathrm{TiO}_{2}$ mean particle size $\left(d_{\text {mean }}\right) 30 \mathrm{~min}$ after mixing for the experimental conditions reported in Table 1 (left side FR $=3 \mathrm{~mL} / \mathrm{min}$ and right side $\mathrm{FR}=110 \mathrm{~mL} / \mathrm{min}$ ).

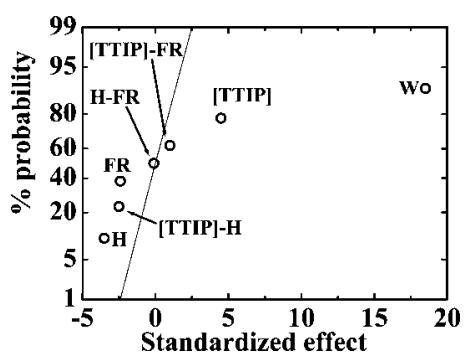

Figure 10. Probability plot for the standardized effects of the investigated parameters and their interactions on $\mathrm{TiO}_{2}$ mean particle size $\left(d_{\text {mean }}\right) 30$ min after mixing; the straight line represents the normal probability distribution.

of each single parameter, by comparing the average response of all experimental runs for which a single parameter is at its low level with the average response of all experimental runs for which the same parameter is at its high level. Moreover this statistical analysis provides additional information on interaction effects and on the relative importance of each effect (and of their interactions).

Effect of Flow Rates on Mixing in the VR. CFD can be used to explore the details of mixing dynamics into chemical reactors and quantify the characteristic mixing time. There exist different strategies to carry out these calculations, and in this work we will adopt the methodology developed in our previous work: ${ }^{27,28}$ however, readers interested in the subject are referred to the specialized literature. ${ }^{29,30}$ Although CFD calculations are affected by several sources of error (e.g., modeling and numerical errors) and a direct and independent validation through comparison with experimental data is always recommended, CFD has now become a mature computational tool that can be safely used to evaluate trends and order of magnitudes of relevant quantities. In this work the flow field in 


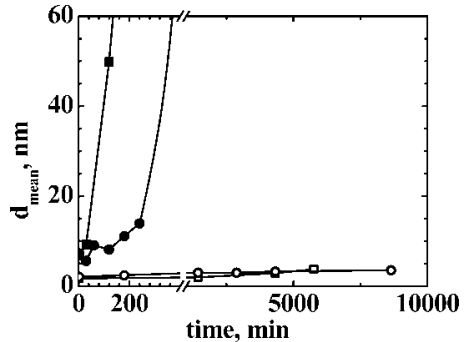

Figure 11. Time evolution of $\mathrm{TiO}_{2}$ mean particle size $\left(d_{\text {mean }}\right)$ for [TTIP] $=$ $0.5 \mathrm{~mol} / \mathrm{L}, H=0.5$ at different hydrolysis ratios $(W)$ and flow rates (FR): (open circles) $W=2$ and $\mathrm{FR}=3 \mathrm{~mL} / \mathrm{min}$; (open squares) $W=2$ and $\mathrm{FR}$ $=110 \mathrm{~mL} / \mathrm{min}$; (filled circles) $W=4$ and $\mathrm{FR}=3 \mathrm{~mL} / \mathrm{min}$; (filled squares) $W=4$ and $\mathrm{FR}=110 \mathrm{~mL} / \mathrm{min}$.

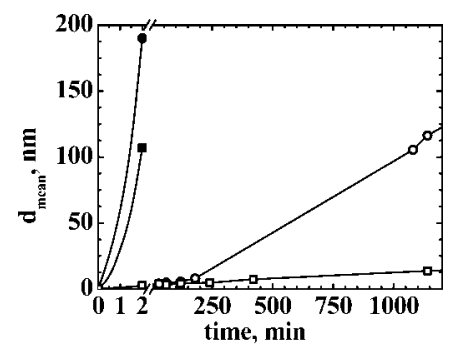

Figure 12. Time evolution of $\mathrm{TiO}_{2}$ mean particle size $\left(d_{\text {mean }}\right)$ for [TTIP] $=$ $1 \mathrm{~mol} / \mathrm{L}, H=0.25$ at different hydrolysis ratios $(W)$ and flow rates (FR): (open circles) $W=2$ and $\mathrm{FR}=3 \mathrm{~mL} / \mathrm{min}$; (open squares) $W=2$ and $\mathrm{FR}$ $=110 \mathrm{~mL} / \mathrm{min}$; (filled circles) $W=4$ and $\mathrm{FR}=3 \mathrm{~mL} / \mathrm{min}$; (filled squares) $W=4$ and $\mathrm{FR}=110 \mathrm{~mL} / \mathrm{min}$.

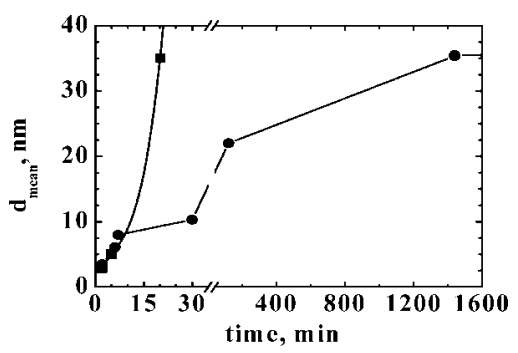

Figure 13. Time evolution of $\mathrm{TiO}_{2}$ mean particle size $\left(\mathrm{d}_{\text {mean }}\right)$ for $[\mathrm{TTIP}]=$ $0.5 \mathrm{~mol} / \mathrm{L}, W=4, \mathrm{FR}=110 \mathrm{~mL} / \mathrm{min}$ and for $H=0.25$ (filled squares) and $H=0.5$ (filled circles).

the VR has been characterized in the range of operating conditions employed during experiments (i.e., FR from 3 to 300 $\mathrm{mL} / \mathrm{min}$ ).

3-dimensional simulations were run testing several computational grids, ranging from 120000 to 400000 cells, in order to find grid independent solutions. The final grid was constituted by about 170000 cells and was refined near the wall and in the proximity of steep velocity gradients. Simulations in turbulent conditions were run using the so-called Reynolds-averaged Navier-Stokes equations approach, by employing the standard $k-\varepsilon$ turbulence model and non-equilibrium wall functions for the near wall treatment. Other turbulence models and near wall treatments were tested, but the use of more sophisticated approaches did not affect the final predictions very much.

The flow field was first simulated assuming that the two reactant solutions were characterized by identical physical properties. In fact, it is important to remember here that in all the experiments the final solid volume fraction, when all the precursor was converted into solid particles, was kept around $0.5-1 \%$. Moreover, the residence time of the solutions in the VR is very short, therefore in most cases when the reacting solution exits the reactor, it contains very small particles, that
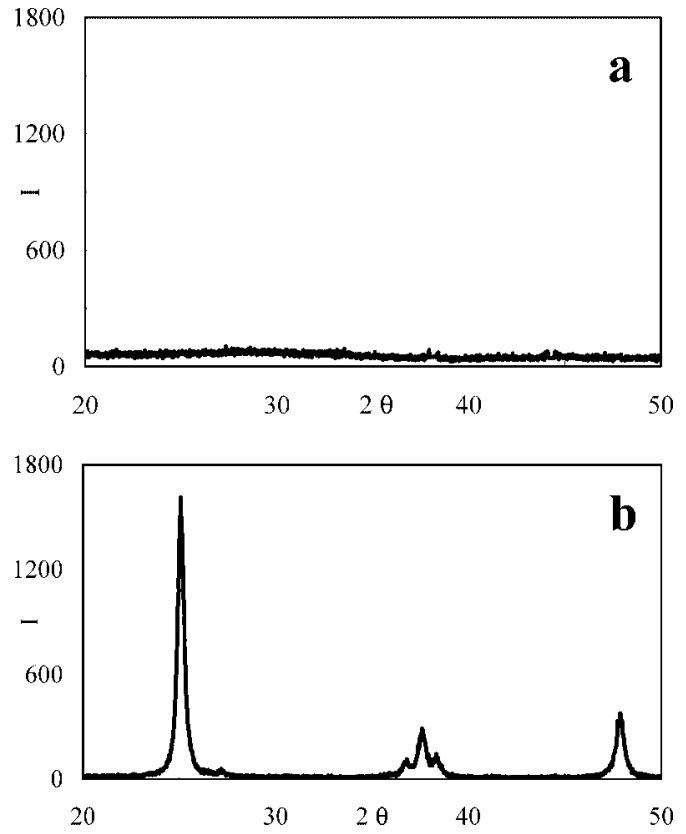

Figure 14. XRD patterns of $\mathrm{TiO}_{2}$ powders after drying and before (a) and after (b) thermal treatment for [TTIP] $=1 \mathrm{~mol} / \mathrm{L}, W=4, H=0.25$, and $\mathrm{FR}=110 \mathrm{~mL} / \mathrm{min}$. For this sample after thermal treatment semiquantitative analysis shows $98 \%$ of anatase and $2 \%$ of rutile.

Table 2. Equivalent Sphere Diameter $\left(d_{\mathrm{BET}}\right)$ Measured before and after Calcination at $400^{\circ} \mathrm{C}$ for $2 \mathrm{~h}$ for Four Different $\mathrm{TiO}_{2}$ Samples Prepared under the Operating Conditions Listed in the First Four Columns

\begin{tabular}{|c|c|c|c|c|c|}
\hline \multicolumn{4}{|c|}{ operating conditions used during synthesis } & \multirow{2}{*}{$\begin{array}{c}\text { before } \\
\text { calcination } \\
d_{\mathrm{BET}}, \mathrm{nm}\end{array}$} & \multirow{2}{*}{$\begin{array}{c}\text { after } \\
\text { calcination } \\
d_{\mathrm{BET}}, \mathrm{nm}\end{array}$} \\
\hline $\begin{array}{c}{[\mathrm{TTIP}],} \\
\mathrm{mol} / \mathrm{L}\end{array}$ & $W$ & $H$ & $\begin{array}{c}\mathrm{F} R, \\
\mathrm{~mL} / \mathrm{min}\end{array}$ & & \\
\hline 1 & 2 & 0.25 & 110 & 5 & 11 \\
\hline 0.5 & 2 & 0.25 & 110 & 4 & 10 \\
\hline 0.5 & 4 & 0.25 & 110 & 5 & 11 \\
\hline 1 & 4 & 0.25 & 110 & 10 & 16 \\
\hline
\end{tabular}

very unlikely affect the liquid phase. It is also important to keep in mind that although water and isopropyl alcohol are completely miscible, the process is characterized by a slow kinetics and therefore it generally takes some time to achieve complete mixing at the molecular level. However in our experiments we are not mixing a solution of pure water with a solution of pure isopropyl alcohol; we are instead mixing a solution of water in isopropyl alcohol and a precursor in isopropyl alcohol. As clearly indicated by some simple visual observations, mixing of these two solutions seems a typical mixing process where two fluids with similar properties (i.e., two solutions of isopropyl alcohol characterized by the same density of $0.785 \mathrm{~kg} / \mathrm{L}$ and a viscosity of $2 \mathrm{cP}$ ) get in intimate contact. The calculated flow field was then used to characterize mixing in the VR. This is generally done by investigating the dispersion in the reactor of an inert tracer that is introduced in one of the two inlets. The theoretical and numerical details of the calculations performed here are beyond the scope of this work, and readers interested on the subject are referred to our previous work. ${ }^{21}$ In Figure 2 the inert tracer average concentration profiles in the VR at three different $\mathrm{FR}$ values $(\mathrm{FR}=10,100$, and $300 \mathrm{~mL} / \mathrm{min})$ are reported. As it is possible to see, the inert tracer normalized averaged concentration $\langle\xi\rangle$ is equal to 1 in one inlet and equal to 0 in the other one. As FR increases, mixing becomes faster and faster guaranteeing more uniform and homogeneous reactant concentrations in the reactor and at the reactor outlet; in fact, with increasing FR more and more regions of the reactor are 


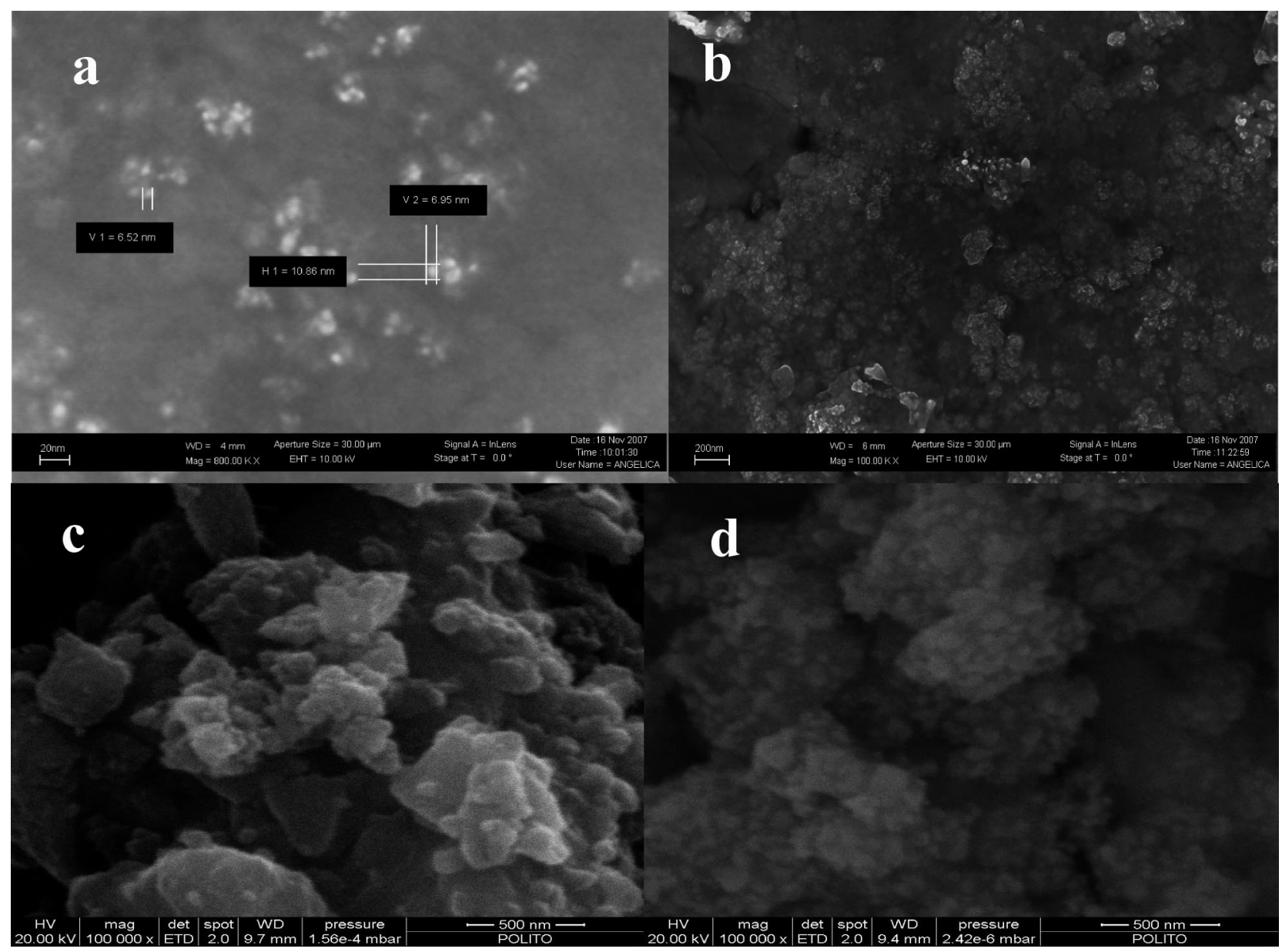

Figure 15. SEM and FE-SEM images of $\mathrm{TiO}_{2}$ samples synthesized at $[\mathrm{TTIP}]=1 \mathrm{~mol} / \mathrm{L}, H=0.25$, and $\mathrm{FR}=110 \mathrm{~mL} / \mathrm{min} ;(\mathrm{a}, \mathrm{b}) \mathrm{W}=2 \mathrm{before}$ calcination; (c) $W=4$ before calcinations; (d) $W=4$ after calcinations.

characterized by a tracer concentration of 0.5 (that corresponds to perfect mixing). The average concentration of the inert tracer however contains information only on the mixing feature at the macroscopic level (or macro-mixing). Since reactions occur at the molecular level, it is important to quantify also their level of segregation at the microscopic level (or micromixing), and this can be done by calculating the so-called intensity of segregation $\left(I_{\mathrm{s}}\right)$. This quantity is equal to 1 for systems perfectly segregated at the molecular level and is equal to 0 for systems perfectly mixed at the molecular level. The contour plots of the intensity of segregation for the three investigated FRs are also reported in Figure 2. Again as it is possible to observe an increase in FR causes an improvement of mixing also at the molecular level resulting in smaller and smaller values of the intensity of segregation.

From these contour plots it is possible to calculate the overall mixing time, namely the time required for a volume of fluid to be completely mixed at the macro- and microscale levels, ${ }^{21,23,24,27,28}$ and in the specialized literature these two steps are usually referred to as macro- and micromixing. It should be reminded here that although the effects on the final product quality of macro- and micromixing are usually different, it is difficult to distinguish among them without more detailed information concerning the kinetics of the particle formation process; therefore in this work only the overall mixing time will be considered. For the three values of FR $(=10,100$, and $300 \mathrm{~mL} /$ min) investigated, the mixing time was found to be equal to 8 , 0.06 , and $0.009 \mathrm{~s}$, respectively.

If these mixing times are compared to the mean residence time for the three FR values (i.e., 3, 0.3 and $0.1 \mathrm{~s}$ ), it is possible to see that for $\mathrm{FR}=10 \mathrm{~mL} / \mathrm{min}$ the mixing time is higher than the residence time, which indicates that the reactants leave the reactor partially unmixed, whereas for the other cases the ratio between the mixing and the mean residence time is significantly smaller than 1 and decreases with increasing FR, indicating that reactants mix faster and faster and leave the reactor completely mixed.

The mixing time is very important since it can be used to scale up a particular process. For example for the process under investigation, if higher flow rates must be processed, a bigger VR could be used, and the proper operating conditions could be found simply by calculating the FR value that results in the same mixing time of the laboratory scale equipment, as shown in our previous work, on a different device. ${ }^{21}$

\section{Experimental Results and Discussion}

The experimental campaign was planned through a DOE with $2^{4}$ runs each repeated twice, resulting in 32 experiments. Particle characterization was carried out in the suspension both as soon as particles were synthesized after mixing the reactant solutions in the VR and by tracking with time the evolution of these particulate systems; further characterization was then carried out on selected samples after filtering, drying, and calcination. The regression analysis was applied both on the data obtained 2 min after mixing and on the measurements carried out at 30 min after mixing.

The typical volume PSDs measured 2 min after mixing for the same experiment repeated twice are reported in Figure 3. The standard deviation for this particular case is about $0.6 \mathrm{~nm}$, 
whereas the averaged standard deviation for all the experiments is a bit smaller, around $0.4 \mathrm{~nm}$. Moreover, as it will become clearer later, for some experiments three or four runs were carried out, illustrating that the synthesis and characterization protocol is very reproducible.

The mean volume particle size $\left(d_{\text {mean }}\right)$ of $\mathrm{TiO}_{2}$ particles measured 2 min after mixing the solutions in the VR are reported in Figure 4 for the operating conditions listed in Table 1. Since the effects of four parameters are studied, results belong to a hypercube of dimension four; however, they are generally displayed geometrically as two cubes. On the left of Figure 4 the effect of [TTIP], $W$, and $H$ is reported at low FR, whereas on the right the effect of the same parameters is reported at high FR. In most cases sols were obtained and the reported mean particle size is that measured with DLS; however, in some cases gels were obtained as soon as the suspension left the reactor, and in these cases the particulate system could not be characterized by a consistent mean particle size (since theoretically a gel is characterized by infinite size).

From these measurements through the ANOVA analysis the main effect of each parameter and their interactions can be calculated. In Figure 5 the main effects of $W$, [TTIP], $H$, and FR on the mean particle size after 2 min are calculated from the statistical regression operated on the raw experimental data. The data reported in Figure 5 represent the results of the regression and interpolation operated along lines passing through the center of the hypercube and moving from the low value of each single parameter to the high value, and these are the average values of the responses of all experiments carried out at low and high levels. In other words, the numbers underlying the four main effect plots do not correspond to any of the 32 experiments: they represent instead the average of many experiments carried at low and high levels of the four parameters. It is probably also useful to anticipate here that the detected trends rose above the experimental variability, as discussed later.

It is possible to observe that an increase of $W$ from 2 to 4 causes an increase in the mean particle size due to the effect of the water content on the competition between hydrolysis and condensation, as already explained in the Introduction and as reported by other authors. ${ }^{11}$ An increase in the mean particle size is also observed when the initial alkoxide concentration [TTIP] is increased from 0.5 to $1 \mathrm{~mol} / \mathrm{L}$ and this is probably related to the fact that higher initial concentrations favor growth of $\mathrm{TiO}_{2}$ particles rather than nucleation of new ones, resulting in bigger $\mathrm{TiO}_{2}$ particles. The effect of the flow rate (FR) goes in the opposite direction, in fact an increase in FR causes the formation of smaller $\mathrm{TiO}_{2}$ particles.

The effect of the FR was explored in a wider range of FR values and for $[\mathrm{TTIP}]=1 \mathrm{~mol} / \mathrm{L}, W=4$, and $H=0.25$, and results are reported in Figure 6, confirming the trend highlighted by the DOE analysis: smaller and smaller $\mathrm{TiO}_{2}$ particles are obtained when the solutions are mixed faster in the VR. All the repetitions of each experimental run are reported in Figure 6 , highlighting the good experimental reproducibility and confirming that this is an actual trend rising above experimental uncertainty. This is a well-known result in many particle formation processes: an increase in the flow rate results in better and faster mixing; that in turn results in higher nucleation rates, producing a numerically denser population of smaller particles. Moreover in Figure 6 two regions are clearly visible, one at FR smaller than $100 \mathrm{~mL} / \mathrm{min}$ where an increase of FR causes a significant reduction of $\mathrm{TiO}_{2}$ mean particle size, and one for FR greater than $100 \mathrm{~mL} / \mathrm{min}$ where an increases in FR does not affect much the final mean particle size. This is related to the fact that particle formation is also characterized by a typical time-scale, and when the characteristic mixing time becomes smaller than the particle formation time-scale no further effect is detected (since mixing is already significantly faster than the chemical reaction). For the particular case under investigation (i.e., $[\mathrm{TTIP}]=0.5 \mathrm{~mol} / \mathrm{L}, W=4$, and $H=0.25$ ) the breakpoint occurs at $\mathrm{FR}=100 \mathrm{~mL} / \mathrm{min}$ resulting in a characteristic particle formation time-scale equal to $0.059 \mathrm{~s}$. Although this information is very important for process scale up, and although the sol-gel literature is quite vast, we did not find any quantitative particle formation kinetics measurement that could confirm this number for the operating conditions investigated in this work.

In Figure 5 the main effect of the acid to alkoxide concentration ratio $\mathrm{H}$ on the mean size of $\mathrm{TiO}_{2}$ particles is also reported and shows only a small increase in the mean particle size. The effect of the acid is 2-fold, in fact, it acts as a hydrolysis catalyst and as an aggregation suppressor. At 2 min the catalytic effect is prevalent, whereas, as it will become clearer later, at $30 \mathrm{~min}$ the aggregation suppressor effect is more important.

The plots of the main effects on the $\mathrm{TiO}_{2}$ mean particle size 2 min after mixing reported in Figure 5 are important because they can also be used to estimate the relative importance of each parameter in the investigated range. In fact, the results reported clearly show that the effects of $W$ and [TTIP] are more important than those of FR that in turn is more important than that of $H$.

However these are just the main effects of the four parameters. As it is well-known the DOE technique is very powerful since it also allows the quantification of the interaction effects of each single parameter when the other three are kept at low and high values. For the case under investigation for each of the 4 parameters there are 3 relevant interaction plots, at low and high levels of the other three, resulting in a total of 12 interaction plots, corresponding to the 12 edges of the hypercube. Usually results are reported in a compact form in six different plots, as shown in Figure 7. For example, the three plots in the first row report the effect of [TTIP], $H$, and FR on the mean particle size for high and low values of $W$, whereas the three plots on the last column report the effect of the FR when $W$, [TTIP], and $H$ are fixed at their low and high values. A detailed comparison of Figures 5 and 7 shows that not only is the main effect of $W$ and [TTIP] important but also that the effect of the FR at high $W$ and/or at high [TTIP] is very important, whereas, for example, the effect of the same parameter at low $W$ and/or low [TTIP] values is much less important. This is probably related to the fact that at high values of $W$ and [TTIP], hydrolysis and condensation are very fast, probably almost instantaneous, and therefore the significant reduction of the mixing time in the VR when the flow rate is changed from 3 to $110 \mathrm{~mL} / \mathrm{min}$ has a strong effect on the reaction path. On the contrary at low values of $W$ and [TTIP], hydrolysis and condensation are very slow, probably much slower than mixing at the two FR values investigated, and therefore in both cases reactants have enough time to properly mix before particle formation occurs.

The relative importance of each main effect (and their interactions) in comparison with the experimental error, estimated through the repetition of each experiment, can be evaluated from the probability plot of the standardized effect, that for the case under investigation is reported in Figure 8. It is important to remind the reader that the influence of a parameter is considered statistically relevant when its standardized effect lies far away from the straight line, representing in this logarithmic plot the normal probability distribution. The 
relevance of each single parameter and their interactions is also estimated through the $p$-value; typically this value is compared against a threshold value, labeled as alpha $(\alpha)$, and equal to 0.05: if the $p$-value is lower than 0.05 , then the effect of the parameter is significant $(95 \%$ confidence factor that the effect is real and not a random fluctuation). For the considered data the effect is statistically significant (if compared with the experimental error estimated from the repetition of each single experiment) for the main effect of $W$, [TTIP], and for the interaction between $W$ and [TTIP] and $W$ and FR.

For all the 32 runs the time evolution of $\mathrm{TiO}_{2}$ particle suspensions was tracked in order to investigate the effect of the operating conditions not only on the characteristics of the immediately synthesized particles but also on their characteristics after some time and on the final product. It is therefore interesting to apply the same statistical analysis on $d_{\text {mean }}$ for the $\mathrm{TiO}_{2}$ particles after $30 \mathrm{~min}$ of gentle stirring since the beginning of the reaction. Results are reported in Figure 9 and as it is possible to see in some cases the sols remained stable and only slightly changed their mean particle size, but in other cases particle agglomeration resulted in the formation of a gel. The statistical analysis of this data set at 30 min mainly confirms the already reported results and can be summarized in the probability plot reported in Figure 10. As it is possible to observe, after $30 \mathrm{~min}$ from mixing the two reactant solutions, the main effects on the mean particle size are the water to alkoxide concentration ratio $W$ and the initial alkoxide concentration [TTIP]. As for the mean particle size at 2 min also after $30 \mathrm{~min}$ the average effect of these two parameters is positive, or in other words an increase in $W$ and [TTIP] results in bigger $\mathrm{TiO}_{2}$ particles. It is moreover very interesting to highlight that for the mean particle size after $30 \mathrm{~min}$ the third most relevant effect is that of the flow rate (FR). As for the mean particle size after $2 \mathrm{~min}$, the average effect is still negative, in fact, the $\mathrm{TiO}_{2}$ particles produced at higher flow rate generally grow slower than those formed at low flow rates. It is also interesting to notice that the effect of the FR is now more important, showing that if its effect is already detectable at $2 \mathrm{~min}$, it is even more evident after $30 \mathrm{~min}$ from mixing the reactant solutions and the beginning of the particle formation process. The fourth relevant effect is due to the interaction between FR and [TTIP], namely the effect of the FR at high initial alkoxide concentration, whereas the last relevant parameter is due to the effect of the acid to alkoxide concentration ratio $(H)$. It is possible to see that this parameter has a quite important negative average effect on the mean particle size measured 30 min after mixing. In fact, the $\mathrm{TiO}_{2}$ particle produced at high $H$ values are generally more stable than those at low $H$ values. This is due to the fact that the acid acts as an inhibitor of condensation, since it generates surface charges on the particles, resulting in stronger repulsion forces and much smaller agglomeration rates. This is demonstrated by $\zeta$-potential measurements (Malvern Nano ZS90) performed on some selected samples, showing a consistent increase of $\zeta$-potential when $H$ is increased from 0.25 to 0.5 .

These results are confirmed by the detailed analysis of the time evolution of the mean particle size of the different samples. For example, in Figure 11 the time evolution of the mean particle size $\left(d_{\text {mean }}\right)$ of $\mathrm{TiO}_{2}$ particles synthesized under [TTIP] $=0.5 \mathrm{~mol} / \mathrm{L}, H=0.5$, and by mixing the solutions at different flow rates $(\mathrm{FR}=3$ and $110 \mathrm{~mL} / \mathrm{min})$ and at two different hydrolysis ratios ( $W=2$ and 4$)$ are reported. We see that when $W=2$ the process of particle growth is quite slow and particles synthesized at different FR show very similar time evolutions.
However when $W=4$ the situation is quite different, in fact, the particle size increases at a much faster rate, probably through reaction limited agglomeration (RLA), resulting in the formation of a gelatinous precipitate, characterized by a very high mean particle size. Although in RLA the rate of particle size growth does not depend on mixing, the FR has a strong influence on the final product characteristics and on the gelling transition since it determines the initial size of the nucleated particles, their number density, and their hydrolyzed state. In fact, as seen in Figure 11, under these operating conditions (i.e., [TTIP] = $0.5 \mathrm{~mol} / \mathrm{L}, W=4, H=0.5)$ suspensions synthesized at different FR values resulted in $\mathrm{TiO}_{2}$ particles with different stabilities that degenerate into gels in different time intervals. Also for these time evolution analyses the repetition of each single test showed very good reproducibility.

If the initial alkoxide concentration is increased to [TTIP] $=$ $1 \mathrm{~mol} / \mathrm{L}$ and the acid content is reduced to $H=0.25$, the $\mathrm{TiO}_{2}$ suspensions showed greater tendency to agglomerate and form gels, as shown by the results reported in Figure 12. We see that the effect of the hydrolysis ratio $W$ is very similar to the previous case: an increase in $W$ accelerates hydrolysis and condensation resulting in faster nucleation of smaller particles that then quickly agglomerate. Because for this case particle formation and evolution kinetics are much faster, the effect of the FR used to mix the reactants is different. In fact, as seen in Figure 12, higher FR values, resulting in faster mixing, produced smaller but more stable $\mathrm{TiO}_{2}$ particles that show a smaller tendency to agglomerate.

It is also interesting to highlight the effect of the acid to alkoxide concentration ratio $H$ on the time evolution of the mean particle size. In fact, although this parameter seems to have little effect on the size of newly formed $\mathrm{TiO}_{2}$ particles, it has a tremendous effect on their surface charge and their stability, as reported in Figure 13. In fact, for $H=0.5$ it is possible to observe the formation of transparent sols, stable for longer times. On the other hand, for $H=0.25$ there is a strong reduction of the time of gelation; in fact, a visible gelatinous precipitate is observed after $30 \mathrm{~min}$ from synthesis.

Particles were filtered, dried, and calcined for further characterization. If the investigated parameters have a strong effect on the particle size distribution, they do not seem to have any effect in the final crystalline form, in fact, after drying and before calcination the powder is mainly amorphous and no distinct XRD pattern is found (see Figure 14a). However, after calcination at $400{ }^{\circ} \mathrm{C}$ for $2 \mathrm{~h}$ the main crystalline form was anatase, as clearly visible from Figure 14b, for all of the investigated cases, although in some cases traces of rutile were observed.

Results reported in Table 2 refer to further characterization conducted on selected samples, whereas in Figure 15 some SEM and FESEM images are reported. Results reported in Table 2 and in Figure 15 show that the final $\mathrm{TiO}_{2}$ particles are agglomerates constituted by primary particles of about 5-15 $\mathrm{nm}$. For all the cases investigated there is a clear correspondence between the initial mean particle size measured with DLS ( $\left.d_{\text {mean }}\right)$ and the final equivalent spherical diameter calculated from the specific surface area $\left(d_{\mathrm{BET}}\right)$. This evidence confirms that in the very early stages of the process, particles are quickly nucleated and grow by the addition of single molecules reaching a characteristic size. That is what is measured by DLS 2 min after mixing. Then $\mathrm{TiO}_{2}$ particles aggregate forming bigger structures that can reach hundreds of nanometers; however, the typical 
size of the primary particles is retained resulting in high specific surface areas.

\section{Conclusions}

In this work the effect of mixing on the production of $\mathrm{TiO}_{2}$ particles via the sol-gel route is quantified and compared with the other relevant operating parameters, namely initial titanium precursor concentration, hydrolysis ratio, and acid concentration. The effect of mixing is studied in a VR whose flow field and mixing features are investigated through CFD. The experimental campaign is planned through a $2^{4}$ factorial design and data are analyzed with standard statistical regression tools.

Results clearly show that all the process parameters investigated play an important role on the final particulate product. Moreover, since hydrolysis and condensation reactions are as fast as mixing, this parameter plays an important role. An improvement in the mixing performances during particle synthesis causes a reduction in the initial size and a change in the hydrolyzed state of the particles, affecting also their subsequent evolution into stable sols or gels by agglomeration.

It is therefore possible to conclude that mixing can be actively used and must be properly controlled in sol-gel processes in order to guarantee the final characteristics of the particulate product under investigation.

The results reported in this work also suggest that it is worthy of future investigations to perform populations balance calculations and direct scale-up experiments to confirm the scale-up rule based on the definition of mixing time proposed in this paper and already implemented and used for other reacting systems.

\section{Acknowledgment}

This research project has been partially supported by the CRT foundation through the Lagrange project.

\section{Literature Cited}

(1) Livage, J. J.; Henry, M.; Sanchez, C. Chemistry of the Sol-Gel Process. Sol. State Ion. 1989, 32-33, 633.

(2) Manzini, I.; Antonioli, G.; Bersani, D.; Lottici, P. P.; Gnappi, G.; Montenero, A. X-ray Absorption Spectroscopy Study of Crystallization Processes in Sol-Gel-Derived $\mathrm{TiO}_{2}$. J. Non-Cryst. Solids 1995, 193, 519.

(3) Livage, J.; Jolivet, J. P.; Tronc, E. Electronic Properties of Mixed Valence Oxide Gels. J. Non-Cryst. Solids 1990, 121, 35.

(4) Barboux-Doeuff, S.; Sanchez, C. Synthesis and Characterization of Titanium Oxide-Based Gels Synthesized from Acetate Modified Titanium Butoxide Precursors. Mater. Res. Bull. 1994, 29, 1.

(5) Bleuzen, A.; Barboux-Doeuff, S.; Flaud, P.; Sanchez, C. Rheological Study of Titanium Oxide-Based Gels. Mater. Res. Bull. 1994, 29, 1223.

(6) Moritz, T.; Benfer, S.; Arki, P.; Tomandl, G. Influence of the Surface Charge on the Permeate Flux in the Dead-End Filtration with Ceramic Membranes. Sep. Purif. Technol. 2001, 25, 1.

(7) Kallala, M.; Sanchez, C.; Cabane, B. SAXS Study of Gelation and Precipitation in Titanium-Based Systems. J. Non-Cryst. Solids 1992, 148, 189.

(8) Babonneau, F.; Sanchez, C.; Livage, J. Spectroscopic Characterization of Sol-Gel Processing. J. Non-Cryst. Solids 1998, 106, 170.
(9) Nabavi, M.; Doeuff, S.; Sanchez, C.; Livage, J. Chemical Modification of Metal Alkoxides by Solvents. A Way to Control Sol-Gel Chemistry. J. Non-Cryst. Solids 1990, 121, 31.

(10) Harris, M. T.; Byers, C. H. Effect of Solvent on the Homogeneous Precipitation of Titania by Titanium Ethoxide Hydrolysis. J. Non-Cryst. Solids 1988, 103, 49.

(11) Kim, K. D.; Kim, H. T. Synthesis of Titanium Dioxide Nanoparticles Using a Continuous Reaction Method. Colloid Surf., A 2002, 207, 263.

(12) Turova, N. Y.; Turevskaya, E. P.; Kessler, V. G.; Yanovskaya, M. I. The Chemistry of Metal Alkoxides; Kluwer Academic Publishers: Norwell, MA, 2002.

(13) Tanaka, K.; Kozuka, H. Sol-gel Preparation and Mechanical Properties of Machinable Cellulose/Silica and Polyvinylpyrrolidone/Silica Composites. J. Sol-Gel Sci. Technol. 2004, 32, 73.

(14) Barringer, E. A.; Bowen, H. K. Formation, Packing, and Sintering of Monodisperse $\mathrm{TiO}_{2}$ Powders. J. Am. Ceram. Soc. 1982, 65, 199.

(15) Jean, J. H.; Goy, D. H.; Ring, T. A. Continuous Production of Narrow-Sized and Unagglomerated $\mathrm{TiO}_{2}$ Powders. Am. Ceram. Soc. Bull. 1987, 66, 1517.

(16) Ogihara, T.; Ikeda, M.; Kato, M.; Mizutani, N. Continuous Processing of Monodispersed Titania Powders. J. Am. Ceram. Soc. 1989, 72, 1598.

(17) Rivallin, M.; Benmani, M.; Kanaev, A.; Gaunand, A. Sol-Gel Reactor with Rapid Micromixing: Modelling and Measurements of Titanium Oxide Nano-Particle Growth. Chem. Eng. Res. Des. 2005, 83, 67.

(18) Takagi, M.; Maki, T.; Miyahara, M.; Mae, K. Production of Titania Nanoparticles by Using a New Microreactor Assembled with Same Axle Dual Pipe. Chem. Eng. J. 2004, 101, 269.

(19) Nagasawa, H.; Tsujiuchi, T.; Maki, T.; Mae, K. Controlling Fine Particle Formation Processes Using a Concentric Microreactor. AIChE J. 2007, 53, 196.

(20) Baffi, G.; Cafiero, L. M.; Chianese, A.; Jachuck, R. J. J. Process Intensification: Precipitation of Barium Sulfate Using a Spinning Disk Reactor. Ind. Eng. Chem. Res. 2002, 41, 5240.

(21) Marchisio, D. L.; Rivautella, L.; Barresi, A. A. Design and Scaleup of Chemical Reactors for Nanoparticle Precipitation. AIChE J. 2006, $52,1877$.

(22) Johnson, B. K. Flash Nanoprecipitation of Organic Actives Via Confined Micromixing and Block Copolymer Stabilization. Ph.D. Thesis, Princeton University, Princeton, 2003.

(23) Gavi, E.; Rivautella, L.; Marchisio, D. L.; Vanni, M.; Barresi, A. A.; Baldi, G. CFD Modelling of Nano-Particle Precipitation in Confined Impinging Jet Reactors. Chem. Eng. Res. Des. 2007, 85, 735.

(24) Gavi, E.; Marchisio, D. L.; Barresi, A. A. On the Importance of Mixing for the Production of Nano-particles. J. Dispersion Sci. Technol. 2008, 29, 548.

(25) Montgomery, D. C. Design and Analysis of Experiments; John Wiley \& Sons: Hoboken, NJ, 2005.

(26) Devore, J. L. Probability and Statistics for Engineering and the Sciences; Duxbury Thomson Learning: Pacific Grove, CA, 2000.

(27) Marchisio, D. L.; Barresi, A. A. CFD Simulation of Mixing and Reaction: The Relevance of the Micro-Mixing Model. Chem. Eng. Sci. 2003, $58,3579$.

(28) Gavi, E.; Marchisio, D. L.; Barresi, A. A. CFD Modelling and Scaleup of Confined Impinging Jet Reactors. Chem. Eng. Sci. 2007, 62, 2228.

(29) Fox, R. O. Computational Models for Turbulent Reacting Flows; Cambridge University Press: Cambridge, U.K., 2003.

(30) Baldyga, J., Bourne, J. R. Turbulent Mixing and Chemical Reactions; Wiley \& Sons: Chichester, U.K., 1999.

Received for review February 6, 2008 Revised manuscript received June 1, 2008 Accepted July 16, 2008

IE800217B 